\title{
Indicações Duvidosas ou Infundadas Observadas no Tratamento com Albumina. Estudo em Hospital Universitário do Estado do Rio de Janeiro*
}

\author{
Doubtful or Unfunded Indications Observed with Albumin Treatment. \\ A Study in a Teaching Hospital in Rio de Janeiro State
}

Felipe A. Magalhães ${ }^{1}$, Luiz Stanislau N. Chini

\section{RESUMO}

JUSTIFICATIVA E OBJETIVOS: Embora faça parte do arsenal clínico há mais de 50 anos, o uso de albumina humana ainda é questão controversa, cuja discussão originou a publicação de inúmeros artigos em periódicos de Medicina. As opiniões e justificativas para o seu uso são conflitantes e só recentemente foram realizados estudos de qualidade, conduzidos para avaliar desfechos mais significativos, como o impacto na mortalidade dos pacientes tratados. Os objetivos deste artigo foram verificar das indicações do uso de albumina, comparar essas indicações com as diretrizes da Agência Nacional de Vigilância Sanitária (ANVISA) de 2004, bem como os resultados de recentes pesquisas científica; e estimar o total das despesas com o produto.

MÉTODO: Análise das justificativas do uso de albumina humana nos 206 pedidos solicitados em 2005, identificados no banco de dados do Hospital Universitário Antonio Pedro (HUAP).

RESULTADOS: Grande quantidade de albumina foi utilizada sem embasamento científico, gerando altos

1. Médico Residente em Clínica Médica do Hospital Adventista Silvestre, Rio de Janeiro, RJ

2. Médico do Hospital Universitário Antônio Pedro, Niterói, RJ

*Recebido do Hospital Universitário Antônio Pedro, Niterói, RJ

Apresentado em 18 de outubro de 2006

Aceito para publicação em 07 de março de 2007

Endereço para correspondência:

Dr. Felipe de Andrade Magalhães

Rua Almirante Salgado, 365/302 - Laranjeiras

22240-170 Rio de Janeiro, RJ

Fones: (021) 2205-5418 / 8136-5418

E-mail: felipe.magalhaes@terra.com.br

(C)Associação de Medicina Intensiva Brasileira, 2007 custos para esse hospital. O impacto sobre a saúde dos pacientes não pôde ser avaliado neste estudo.

CONCLUSÕES: Apesar da grande discussão e da publicação de inúmeros artigos sobre o tema, a albumina humana é usada de forma ampla e indiscriminada nesse hospital de ensino.

Unitermos: albumina; Agência Nacional de Vigilância Sanitária (ANVISA); despesa médica; hospital de ensino

\section{SUMMARY}

BACKGROUND AND OBJECTIVES: Although has been available for clinical administration for over 50 years, the use of human albumin keep being a controversial and hotly debated and it is theme of many papers in medical journals. The opinions and justifications for the use of albumin are huge and only more recently were published betters papers leaded to appraisal more significant outcomes as the impact in the mortality of the treat patients. The objectives of this paper are: to check the albumin indications, to compare them with Agência Nacional de Vigilância Sanitária (ANVISA) guideline of 2004, recent scientific researches and to estimate the total cost of the product.

METHODS: Analysis of the justifications of human albumin use in 206 requests, of 2005, identified in Hospital Universitário Antonio Pedro (HUAP) data base.

RESULTS: A great amount of albumin was used without scientific justifications, generating financial expenditures of thousand of reais, but whose impact on the health of the patients is impossible to be evaluated in this work.

CONCLUSIONS: Although the great discussion and many papers about this theme, the albumin is still use in an intense and unrestricted way in this teaching hospital.

Key Words: Agência Nacional de Vigilância Sanitária (ANVISA), albumin, cost, teaching hospital 


\section{INTRODUÇÃO}

A albumina humana disponível para uso clínico desde a década de 1940 nos EUA ${ }^{1}$ é classicamente utilizada para repor o volume intravascular e aumentar a pressão coloidosmótica em diversas situações. No entanto, desde 1998 o seu uso em larga escala sofreu um revés com a publicação de uma metanálise do grupo Cochrane $^{2}$ questionando este uso. Esse artigo apresentou um desenho retrospectivo, no qual foram analisados 30 estudos aleatórios e controlados, que incluíram 1.419 pacientes. $O$ artigo concluiu que a reposição de albumina, quando comparada a de cristalóides ou a nenhuma reposição, gerou mortalidade adicional de seis em cada 100 pacientes com hipovolemia, queimaduras ou hipoalbuminemia.

Embora possa ser identificado o esforço da indústria farmacêutica e de alguns pesquisadores para encontrar argumentos e evidências que justifiquem o seu uso $^{3}$, o influente setor produtor de albumina também não conseguiu produzir artigos de peso para contrapor às crescentes críticas ao seu emprego indiscriminado. O mais famoso e poderoso estudo envolvendo a reposição de albumina, o SAFE, limitou-se a provar que na fase de expansão volêmica da sepse a albumina apresenta taxa de mortalidade estatisticamente igual ao uso de solução fisiológica ${ }^{4}$. Sedrakyan e col. ${ }^{5}$ sugeriram que a albumina diminuiria a mortalidade de pacientes após intervenção cirúrgica de revascularização do miocárdio. Como esse estudo utilizou como grupo controle, a reposição de outros colóides, somente foi possível concluir que, entre os colóides, a albumina é o melhor, mas não que ela diminua a mortalidade como sugeriu o estudo.

Seguindo tendência internacional de produzir recomendações no sentido de evitar o uso indiscriminado de albumina ${ }^{6-8}$, a Agência Nacional de Vigilância Sanitária (ANVISA) publicou a Resolução RDC $n^{\circ} 115$, em 10 de maio de 2004. As diretrizes da ANVISA sobre o uso albumina tomaram por base os estudos publicados até o final de 2003 e o alto custo do produto. Depois dessa data, poucos trabalhos publicados modificaram a essência das indicações fundamentadas na Resolução RDC $n^{\circ} 115$. A despeito dos aspectos positivos da resolução da ANVISA, que propõe uma espécie de padronização do uso da albumina, ainda há espaço para críticas às suas escolhas.

Não obstante, a ANVISA definir como indicação formal a reposição de albumina após as primeiras 48 horas no tratamento dos grandes queimados, o artigo SOAP demonstrou que nesse grupo o uso da albumina levou ao aumento da mortalidade, como outros artigos já haviam inclusive demonstrado que a albumina era inferior ao emprego de solução fisiológica para o restabelecimento hemodinâmico dos pacientes ${ }^{10}$.

A ANVISA não indica a albumina para reposição por perdas agudas, possivelmente dada a ausência de estudos favoráveis e em decorrência do seu alto custo. Nesse caso, os resultados do estudo SAFE ${ }^{4}$, afora a grande casuística, não conseguiram demonstrar diferenças estatísticas na mortalidade, quando comparado o uso de albumina com o de solução fisiológica. Deste modo, é provável que esta orientação da ANVISA tenha sido uma escolha acertada, em especial quando se considera o custo do produto para o sistema de saúde brasileiro (SUS).

A reposição de albumina em pacientes com hipoalbuminemia é o tema que mais tem gerado discussão e foi o grande alvo do artigo do grupo Cochrane ${ }^{2}$. Posteriormente, em outro grande estudo, o SOAP, demonstrou o aumento da mortalidade ${ }^{11}$. Tal pesquisa apresentou resultados muito semelhantes aos do grupo Cochrane em relação ao uso de albumina, mas tem a vantagem de ser um estudo prospectivo, multicêntrico, mesmo que observacional. A posição da ANVISA coincide com tais estudos e define como não recomendado o uso de albumina nesse caso.

Em contraposição a esses estudos, um trabalho de 2004 demonstrou menor morbidade ${ }^{13}$. Entretanto, o estudo apresentou um desfecho primário muito frágil, um grupo de indicações de albumina muito amplo (não foram feitas restrições à indicação, sendo adicionadas todas para a conclusão final), além de incluir estudos muito heterogêneos, alguns produzidos nas décadas de 1950 e 1960, e contabilizar na análise final mais de 17 estudos anteriores à década de 1980. É interessante registrar que tal estudo foi capitaneado por JeanLouis Vincent, o mesmo pesquisador que publicaria o SOAP no ano seguinte ${ }^{11}$. Atualmente, a maioria dos autores considera que o emprego de albumina só para corrigir uma hipoalbuminemia é inadequado ou, nas palavras de Allison e Lobo ${ }^{12}$, uma indicação essencialmente ingênua.

A ANVISA defende o uso de albumina para prevenir a síndrome da hiperestimulação ovariana. Contudo, um ensaio clínico publicado antes mesmo da Resolução RDC n 115 colocou a indicação, no mínimo, em dúvida ${ }^{13}$.

Não há nas diretrizes da ANVISA qualquer recomen- 
dação específica em relação à hipotensão durante a diálise. Adiantando os resultados do presente estudo, todavia, este foi um importante grupo de indicação no HUAP. Um estudo de 2004, comparando soluções fisiológicas com albumina a $5 \%$ para tratamento da hipotensão durante a diálise, não encontrou diferença nos resultados finais e recomendou solução fisiológica como o fluido de escolha ${ }^{14}$.

No tratamento de pacientes cirróticos, ou com ascite, a ANVISA indica a albumina somente para os grandes edemas, que colocam em risco a vida dos pacientes, e para as paracenteses de grande volume. Por sua vez, se a Agência governamental não faz menção à profilaxia de peritonites bacterianas espontâneas (PBE), um ensaio clínico ${ }^{15}$, demonstrou que nos pacientes com cirrose e peritonite bacteriana espontânea o uso simultâneo de albumina e antibiótico é mais eficaz do que apenas antibiótico.

O quadro de SARA não é comentado pela Resolução RDC $n^{\circ} 115$, condição que pode apresentar melhora hemodinâmica com o uso de albumina associada à furosemida. No entanto, a mortalidade ou os resultados em longo prazo não são alterados pelo fármaco ${ }^{16}$.

Diante das poucas indicações com respaldo na literatura científica para o uso de albumina humana, este estudo objetivou identificar e avaliar as suas indicações na população adulta do HUAP, no ano de 2005; compará-las com as indicações da diretriz da Resolução RDC $n^{\circ} 115$ da ANVISA, de 10 de maio de 2004, bem como aos resultados de recentes pesquisas científicas e estimar as despesas com o produto.

\section{MÉTODO}

O Hospital Universitário Antônio Pedro é um hospital de ensino de nível terciário que possui cerca de 400 leitos, emergência aberta ao público e atende exclusivamente pelo Sistema Único de Saúde (SUS). No HUAP todos os pedidos de albumina humana são, obrigatoriamente, solicitados por meio do preenchimento da "solicitação de medicamentos excepcionais" e há uma única forma de apresentação: solução de albumina a $20 \%$ em frascos de $50 \mathrm{~mL}$.

A fim de identificar as indicações para o uso de albumina em 2005, todos os impressos relativos aos 12 meses foram revistos junto à farmácia do HUAP. Os impressos foram subdivididos de acordo com as justificativas de uso e classificados de duas maneiras: segundo as diretrizes da ANVISA na Resolução RDC $n^{\circ}$ 115 e de acordo com o Serviço que prescreveu. Foram ignorados os nomes dos profissionais que prescreveram o produto e dos pacientes tratados, além de terem sido excluídas duas prescrições por falta da data de solicitação e outras 17 prescrições de pacientes pediátricos. Este não é um estudo experimental, não foi acessado nenhum dado visando identificar pacientes e está de acordo com a Declaração de Helsinque.

\section{RESULTADOS}

Foram localizados 206 pedidos de utilização de albumina humana no período de janeiro a dezembro de 2005, correspondentes a 21 serviços médicos do HUAP (Tabela 1). Os pedidos de albumina totalizaram um consumo de 2.890 frascos (Tabela 2), porém 12 pedidos não foram contabilizados por não haver previsão do número de frascos no pedido. Separou-se o número de pedidos por Serviço (Tabela 1) e também o número de frascos solicitados por cada Serviço (Tabela 2). A Unidade de Terapia Intensiva, seguida pela Emergência e a Cirurgia Geral II, foram os serviços que mais consumiram albumina no período estudado. A análise individual das indicações de albumina foi muito difícil de ser realizada por problemas decorrentes do preenchimento do formulário, mas foi possível reunir as indicações em 21 grupos (Tabela 3).

\begin{tabular}{lc} 
Tabela 1 - Número de Pedidos por Serviço & \\
\hline Serviços & Pedidos \\
\hline Unidade de Terapia Intensiva & 31 \\
Emergência & 25 \\
Cirurgia geral II & 18 \\
Doenças infecto-parasitárias & 17 \\
Gastroenterologia & 16 \\
Unidade coronariana & 15 \\
Cirurgia geral I & 13 \\
Clínica médica feminina & 9 \\
Neurologia & 8 \\
Cirurgia vascular & 6 \\
Repouso & 6 \\
Nefrologia e centro de diálise & 5 \\
Cirurgia plástica & 5 \\
Neurocirurgia & 5 \\
Anestesia/recuperação pós-anestésica & 5 \\
Hematologia & 4 \\
Pneumologia & 3 \\
Ortopedia & 2 \\
Cardiologia & 10 \\
Ginecologia & 1 \\
Clínica médica masculina & 1 \\
Sem indicação do serviço & \\
Total & 1 \\
\hline
\end{tabular}


Tabela 2 - Frascos de Albumina Solicitados por Serviço

\begin{tabular}{lc}
\hline Serviços & Frascos \\
\hline Unidade de Terapia Intensiva & 523 \\
Emergência & 316 \\
Cirurgia geral II & 303 \\
Doenças infecto-parasitárias & 267 \\
Gastroenterologia & 219 \\
Unidade coronariana & 208 \\
Cirurgia geral I & 179 \\
Neurologia & 150 \\
Nefrologia e centro de diálise & 110 \\
Clínica médica feminina & 93 \\
Cirurgia vascular & 91 \\
Cirurgia plástica & 72 \\
Neurocirurgia & 44 \\
Anestesia/recuperação pós-anestésica & 42 \\
Hematologia & 31 \\
Clínica médica masculina & 30 \\
Ortopedia & 19 \\
Repouso & 16 \\
Cardiologia & 15 \\
Ginecologia & 12 \\
Pneumologia & 3 \\
Sem indicação do serviço & 147 \\
Total & 2.890 \\
\hline
\end{tabular}

Nota: Encontrado um pedido sem previsão da quantidade de frascos nos serviços de Cirurgia Geral II, Nefrologia, Cirurgia Plástica e Hematologia, e dois pedidos sem a mesma previsão nos serviços de Gastroenterologia, Pneumologia, Anestesiologia e Neurologia.

Tabela 3 - Indicações Utilizadas nos Pedidos de Albumina

\begin{tabular}{lc}
\hline Indicações & No $^{\circ}$ de Pedidos \\
\hline Hipoalbuminemia ou hipoalbuminemia e ede- & 58 \\
ma periférico & \\
Sem justificativa & 46 \\
Hipoalbuminemia e anasarca & 22 \\
Choque séptico & 14 \\
Cirrose hepática e hipoalbuminemia & 10 \\
Cirrose hepática e insuficiência renal aguda & 6 \\
Hipoalbuminemia e desnutrição & 5 \\
Pós-operatórios diversos sem outra justificativa & 5 \\
Grande queimado & 4 \\
Hipotensão durante hemodiálise & 4 \\
Justificativa não legível & 3 \\
Paracentese de grande volume & 3 \\
Paracentese de volume não especificado & 2 \\
Proteinúria & 2 \\
Sepse grave & 2 \\
Hipoalbuminemia e oligúria & 2 \\
Peritonite Bacteriana espontânea & 2 \\
Cirrose e ascite refratária & 2 \\
Síndrome da angústia respiratória do adulto & 1 \\
Insuficiência renal aguda pré-renal & 1 \\
Outras indicações* & 11 \\
Total & 206 \\
\hline
\end{tabular}

*Obs. Nenhuma das indicações desse grupo tem recomendação da ANVISA ou de grandes estudos publicados.

\section{DISCUSSÃO}

Dentre as indicações registradas (Tabela 3), a mais freqüente foi por hipoalbuminemia, com ou sem edema periférico. Entretanto, esta indicação já não é mais defendida por nenhum dos autores de estudos recentes sobre o tema e tampouco pela ANVISA. Em segundo lugar ficaram os pedidos sem qualquer justificativa e, logo a seguir, os pedidos para pacientes com hipoalbuminemia e anasarca ou com derrame cavitário (pleural, pericárdio ou ascite), o que, a rigor, só é indicação válida se o derrame colocar em risco a vida do paciente. Contudo, tal informação não consta de nenhuma das justificativas encontradas nos formulários.

Neste hospital foram consumidos 2.890 frascos de albumina humana que, utilizando os preços publicados pela Revista da Associação Brasileira do Comércio Farmacêutico (edição de dezembro de 2005), totalizaram uma despesa entre $R \$ 585.060,00$ e $R \$$ $727.080,00$, quando considerado o menor e o maior preço praticado no mercado.

\section{CONCLUSÃO}

Entre os pedidos registrados na farmácia do HUAP no ano de 2005, apenas quatro grupos obedeceram aos critérios de indicação formal da ANVISA. Juntos eles totalizaram 11 indicações que corresponderam a, aproximadamente, 5,34\% dos 206 pedidos identificados. Em suma, a grande maioria dos pedidos para a reposição de albumina não tem embasamento científico e nem consta das indicações da Resolução n 115 da ANVISA. Apesar de não poder ser feita uma extrapolação dos dados do estudo Cochrane ${ }^{2}$, que adicionaria seis mortes para cada 100 pacientes tratados, pode-se concluir que algumas centenas de milhares de reais foram desperdiçadas no HUAP devido à indicação inadequada de albumina em 2005. Além do alto custo do produto, que aumenta significativamente as despesas do hospital de ensino, o uso de albumina sem respaldo científico pode ocasionar riscos à saúde dos pacientes.

Sugere-se a publicação de novos estudos demonstrando claramente os benefícios com a reposição de albumina, que o seu uso deva ser desestimulado na maioria das situações clínicas identificadas neste estudo. 


\section{MAGALHÃES E CHINI}

\section{REFERÊNCIAS}

01. Martin GS - Pharmacological aspects of albumin as a niche product in the intensive care unit. Crit Care Med, 2005;33:1667-1669.

02. Human albumin administration in critically ill patients: systematic review of randomised controlled trials. Cochrane Injuries Group Albumin Reviewers. BMJ, 1998;317:235-240.

03. Yamey G - Albumin industry launches global promotion. BMJ, 2000;320:533.

04. Finfer S, Bellomo R, Boyce $\mathrm{N}$ et al - A comparison of albumin and saline for fluid resuscitation in the intensive care unit. $\mathrm{N}$ Engl $\mathrm{J}$ Med, 2004;350:2247-2256.

05. Sedrakyan A, Gondek K, Paltiel D et al - Volume expansion with albumin decreases mortality after coronary artery bypass graft surgery. Chest, 2003;123:1853-1857.

06. Expert Working Party - Report of the expert working party of the committee on safety of medicines. 1999. http://www.open.gov.uk/mca/albumin1.htm.

07. Evidence-based colloid use in the critically ill: American Thoracic Society Consensus Statement. Am J Respir Crit Care Med, 2004;170:1247-1259.

08. Indications for the use of human albumin solutions: an expert report. Human albumin expert group. Schweiz Med Wochenschr, 2000;130:516-522.
09. http://e-legis.anvisa.gov.br/leisref/public/showAct.php?id=11025. Acesso em 11/09/2006.

10. Gore DC, Dalton JM, Gehr TW - Colloid infusions reduce glomerular filtration in resuscitated burn victims. J Trauma, 1996;40:356-360.

11. Vincent JL, Sakr Y, Reinhart K et al - Is albumin administration in the acutely ill associated with increased mortality? Results of the SOAP study. Crit Care, 2005;9:R745-R754.

12. Allison SP, Lobo DN - Debate: Albumin administration should not be avoided. Crit Care, 2000;4:147-150.

13. Bellver J, Munoz EA, Ballesteros A et al - Intravenous albumin did not prevent moderate-severe ovarian hyperstimulation syndrome in high-risk IVF patients: a randomized controlled study. Hum Reprod, 2003;18:22832288

14. Knoll GA, Grabowski JA, Dervin GF et al - Randomized, controlled trial of albumin versus saline for the treatment of intradialytic hypotension. $J$ Am Soc Nephrol, 2004;15:487-492.

15. Sort $P$, Navasa M, Arroyo $V$ et al - Effect of intravenous albumin on renal impairment and mortality in patients with cirrhosis and spontaneous bacterial peritonitis. N Engl J Med, 1999;341:403-409.

16. Martin GS, Moss M, Wheeler AP et al - A randomized, controlled trial of furosemide with or without albumin in hypoproteinemic patients with acute lung injury. Crit Care Med, 2005;33:1681-1687. 